\title{
Metanephric adenoma: A report of two cases and review of the literature
}

\author{
YONGQING LAI ${ }^{1,2}$, DUQUN CHEN ${ }^{1,2,3}$, XIAONAN XU ${ }^{1,2,4}$, ZUHU YU $^{1,2}$, \\ LIANGCHAO NI ${ }^{1,2}$, SHANGQI YANG ${ }^{1,2}$ and $\mathrm{YUN} \mathrm{CHEN}^{5}$
}

\author{
${ }^{1}$ Department of Urology, Peking University Shenzhen Hospital; ${ }^{2}$ The Guangdong and Shenzhen Key Laboratory of Male \\ Reproductive Medicine and Genetics, Institute of Urology, Peking University Shenzhen Hospital, \\ Shenzhen PKU-HKUST Medical Center, Shenzhen, Guangdong 518036; ${ }^{3}$ Anhui Medical University, Hefei, Anhui 230032; \\ ${ }^{4}$ Shantou University Medical College, Shantou, Guangdong 515041; ${ }^{5}$ Department of Ultrasound Division, \\ Peking University Shenzhen Hospital, Shenzhen, Guangdong 518036, P.R. China
}

Received May 25, 2013; Accepted September 9, 2013

DOI: $10.3892 / \mathrm{mco} .2013 .184$

\begin{abstract}
Metanephric adenoma (MA) is a rare benign renal neoplasm of originating in the epithelial cells, which is often difficult to distinguish from malignant neoplasms preoperatively. In this study we present two cases of patients with MA with the aim to analyze the clinical manifestations, imaging, pathology, diagnosis and treatment of MA. Computed tomography (CT) of the first case revealed a solid well-demarcated tumor, $5.5 \times 5.4 \mathrm{~cm}$ in diameter. Ultrasound examination of the second patient revealed a hypoechoic mass with solid aspect and irregular limits in the upper pole of the right kidney. CT confirmed the presence of a $4 \mathrm{~cm}$ expansive tumoral formation with intermediate attenuation and minimum venous contrast enhancement at that anatomic site. The two cases underwent surgery and the patients were free from recurrence after a follow-up period of 18 months to 2 years. We also present a supplementary review of previously published cases and related literature.
\end{abstract}

\section{Introduction}

Metanephric adenoma (MA) is a rare renal neoplasm, accounting for $0.2 \%$ of adult renal epithelial neoplasms (1). It is usually benign and was first described by Brisigotti et al (2). The majority of MAs occur in patients 50-60 years of age with a female:male ratio of 2:1 (3). However, a case of a MA in a 7-year-old female has also been reported (4). According to the 2004 WHO Classification of Renal Cell Tumors, MA was commonly described as a unilateral lesion, although a

Correspondence to: Professor Yongqing Lai, Department of Urology, Peking University Shenzhen Hospital, Institute of Urology of Shenzhen PKU-HKUST Medical Center, 1120 Lianhua Road, Shenzhen, Guangdong 518036, P.R. China

E-mail: yqlord@163.com

Key words: metanephric adenoma, case report multifocal case of childhood MA has been reported (5). The clinical and radiological characteristics of MA are non-specific and a histopathological examination is required in order to establish a definitive diagnosis. Partial nephrectomy should be considered as a therapeutic option and regular follow-up is required to detect late recurrences. In this study we present two patients with MA and a supplementary review of previously published cases and related literature.

\section{Case reports}

A 35-year-old female was admitted to Peking University Shenzhen Hospital (Shenzen, China) with an incidental finding of a right flank mass 1 week earlier. The hemoglobin level was $146 \mathrm{~g} / \mathrm{l}$, the red blood cell count was $5.31 \times 10^{12} / \mathrm{l}$ and other routine hematological examinations and biochemical tests were within normal limits. Computed tomography (CT) revealed a solid tumor in the upper pole of the right kidney, measuring $5.5 \times 5.4 \mathrm{~cm}$. The tumor was well-demarcated, $44 \mathrm{HU}$ and marginally enhanced following contrast enhancement and was characterized by septation and cystic areas in its interior. Considering the size of the mass and the possibility of malignancy, a radical nephrectomy was performed. Microscopically, the excised tumor exhibited characteristically monotonous, small, acinar and tubular structures lined by small, uniform epithelial cells with scanty cytoplasm and hyperchromatic round nuclei. Based on the histolological and cytogenetic characteristics, a diagnosis of MA was suggested. The patient was free from recurrence after 2 years of follow-up.

The second patient was a 46-year-old female with no previous health problems, who presented with flank pain persisting for 2 months and was admitted to Peking University Shenzhen Hospital. The hemoglobin level was $109 \mathrm{~g} / \mathrm{l}$ and the red blood cell count was $5.34 \times 10^{12} / 1$. The ultrasound examination revealed a hypoechoic mass with a solid aspect and irregular limits in the upper pole of the right kidney. CT confirmed the presence of a $4-\mathrm{cm}$ expansive tumoral formation with intermediate attenuation and minimal venous contrast enhancement at that anatomical site. The patient was subjected to a successful partial nephrectomy by open surgery. 


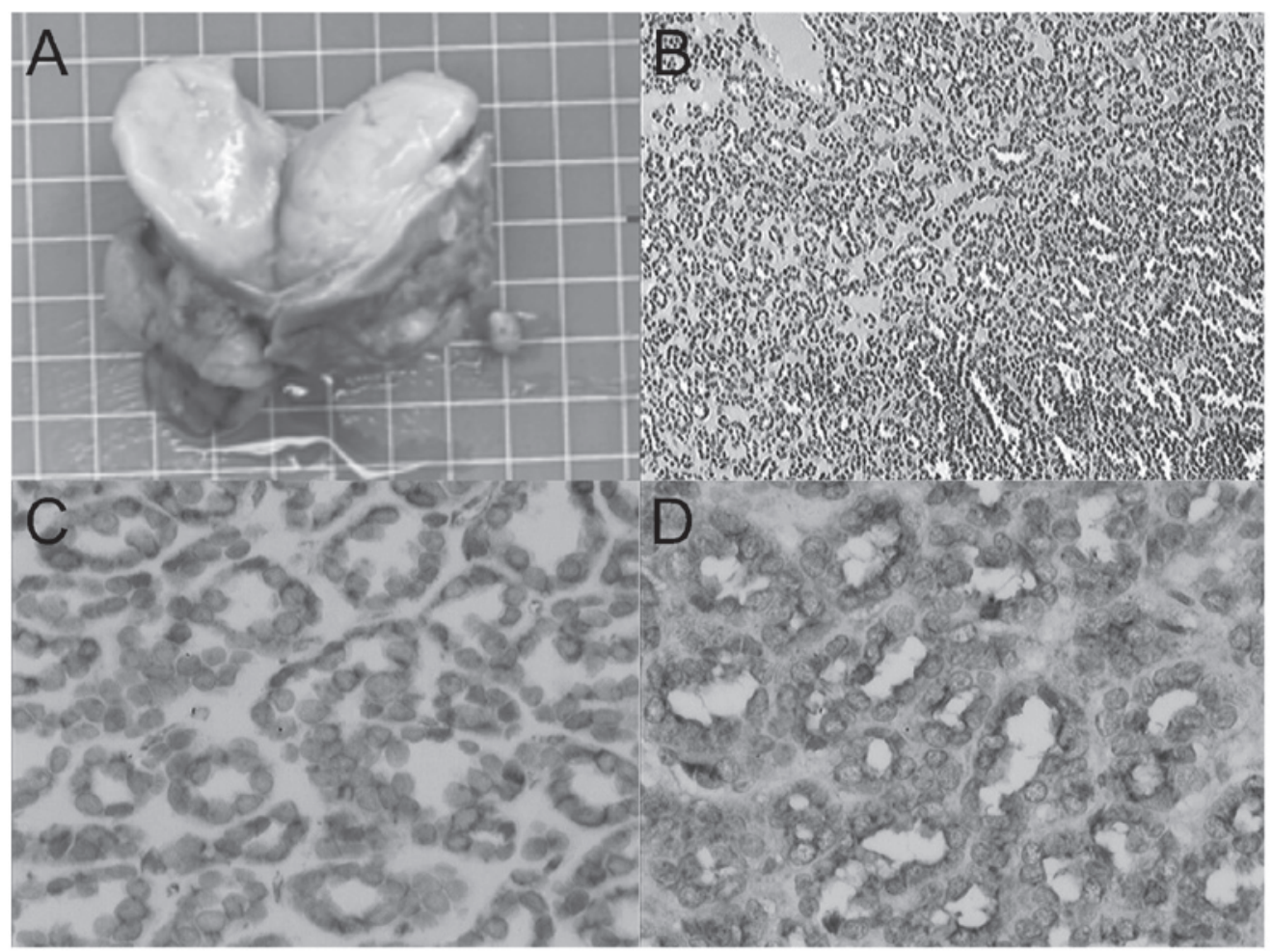

Figure 1. Metanephric adenoma of the second patient. (A) Macroscopic appearance of the tumor. (B) Retiform and micropapillary architecture of the tumor (hematoxylin and eosin staining; magnification, x100). (C) Diffuse, strong positive cytoplasmic immunostaining of the tumor for CD57 (magnification, x200). (D) Diffuse, strong positive cytoplasmic immunostaining of the tumor for vimentin (magnification, $\mathrm{x} 200$ ).

Microscopically, the tumor was composed of tightly packed, uniform, small epithelial cells with small regular nuclei, a high nucleus-to-cytoplasm ratio and the absence of mitotic figures. Immunohistochemically, the tumor exhibited diffuse positive staining for CD57 and vimentin and was only focally and weakly positive for Wilm's tumor 1 (WT1). Immunostaining for epithelial membrane antigen (EMA), CD10 and CD34 was negative in the tumor. The findings were compatible with a diagnosis of MA (Fig. 1). The patient remains disease-free 18 months following the surgery.

\section{Discussion}

Approximately 50\% of MAs are not clinically symptomatic, although flank pain, haematuria, fever, hypertension and polycythaemia have been reported. Among renal lesions, MA is associated with the highest incidence $(10 \%)$ of polycythemia (6), which may be attributed to the paraneoplastic syndrome. Yoshioka et al (7) detected high concentrations of erythropoietin, granulocyte macrophage colony-stimulating factor, granulocyte colony-stimulating factor, interleukin (IL)-5 and IL-8 in the cell culture medium of MA. However, futher evidence is required in order to establish an association between polycythemia and these biomolecules. In addition, hypercalcemia and chyluria were reported in rare cases (8).

The imaging characteristics of the lesions have not been definitively described. On sonography, MA more commonly presents as a well-circumscribed, round or oval, hypo- or hyperechoic solid mass, occasionally with a hypoechoic rim, and may appear as a fluid-containing mass. The power Doppler evaluation demonstrated that this type of lesion is hypovascular. In detail, MA appears as an iso- or hyperdense mass in relation to adjacent renal parenchyma on pre-contrast $\mathrm{CT}$, with minimal enhancement on contrast CT. A case reported by Jinzaki et al (9) exhibited peak enhancement of the neoplasm in the late nephrographic phase. On magnetic resonance imaging, MA appears as an isointense or hypointense mass on T1-weighted images and as a heterogeneous hyperintense mass on T2-weighted images.

Pathological examination of the surgical specimen revealed a firm, light brown lesion with a reticulated central area and clear delimitation from the adjacent parenchyma. Unlike renal adenoma, which is by definition $<5 \mathrm{~mm}$ in diameter, the diameter of MA ranges from 6-200 $\mathrm{mm}$. The renal tumors presented in this study were 40 and $55 \mathrm{~mm}$ in diameter. Microscopically, the tumor is composed of small, uniform epithelial cells with scant cytoplasm and hyperchromatic round nuclei, that form tubular or glomerular-like structures. Abundant psammoma bodies are commonly present, while mitotic figures are absent. Large tumors may appear as heterogeneous, hypovascular masses with frequent foci of hemorrhage, necrosis and calcifications.

Despite the absence of consistent immunohistochemical staining patterns, there is frequently focal positivity for CD7 and CD57, diffuse positivity for vimentin, WT1 and AE1/AE3 and negativity for EMA, S-100, carcinoembryonic antigen, chromogranin A, synaptophysin, actin and $\alpha$-methylacyl-CoA racemase (10). This marker profile may be used in the differential diagnosis of papillary renal cell carcinoma (PRCC), since PRCC is positive for EMA and CK7 (11).

MA has been linked to PRCC due to certain overlaps of histological characteristics and common molecular 
alterations, such as the gains of chromosomes 7 and 17 and loss of gender chromosomes. However, MA exhibits no duplication of chromosomes 7 and 17q21.32, which is a consistent molecular characteristic of PRCC. A previous case report described a balanced translocation $[\mathrm{t}(9 ; 15)(\mathrm{p} 24 ; \mathrm{q} 24)]$ and a balanced paracentric inversion of chromosome 12 [inv(12) (q13q15)] (12).

Fine-needle aspiration may be used as a less invasive method for the diagnosis of MA, although it is less accurate than nephrectomy. Additionally, cytological diagnosis using fine-needle aspiration may be challenging (10).

The differential diagnosis of renal MA includes PRCC and epithelial Wilm's tumor (WT). PRCC accounts for 7-15\% of renal cell cancer cases. Histologically, the tumor cells are arranged in an entirely papillary pattern with fibrovascular cores. The tumor cells have abundant oncocytic cytoplasm and exhibit stratification or pseudostratification. The nuclei are large and hyperchromatic, with distinct nucleoli. Mitotic figures are evident. Scattered psammoma bodies, Prussian blue-positive hemosiderin granules and pale foamy cells are observed. Trisomies of the chromosomes 7 and 17 and loss of gender chromosomes may also be detected by genetic studies. WTs are three-phase embryonic renal tumors consisting of varying amounts of blastemic, epithelial and mesenchymal structures, with a low incidence rate in adults. MA may be difficult to distinguish from epithelial (tubular predominant) type Wilms' tumor, although the latter grows quickly and exhibits abundant mitotic figures with distinct cellular atypia.

MA is an invariably benign renal tumor with a favorable prognosis. However, the majority of reported cases of MA were subjected to nephrectomy due to the difficult preoperative differentiation from malignant renal tumors. The biological behavior of MA is benign and is always accompanied by a successful outcome with nephrectomy or mass resection. However, a case of MA with metastases in the periaortic, hilar and aortic bifurcation lymph nodes was reported in a 7-year-old female (13) and a case of MA containing foci of papillary carcinoma and a regional metastatic lymph node was reported in an 11-year-old female (14). Furthermore, bone metastases in adults have been described (15).

In conclusion, MA is a rare, benign neoplasm of epithelial cells, which is complicated to preoperatively distinguish from other malignant neoplasms. In this study we present the cases of two patients with MA. The two patients underwent surgery and the pathological findings indicated that MA was the correct diagnosis. The two patients were free of recurrence following longterm follow-up. Given the rarity of this tumor and the lack of highly predictive clinical or radiographic criteria, we suggest that a histopathological examination is necessary in order to establish a definitive diagnosis.

\section{Acknowledgements}

This study was supported by the National Natural Science Foundation of China (Grant No. 81101922), the Medical Scientific Research Foundation of Guangdong Province of China (Grant Nos. A2012584 and A2013606) and the Science and Technology Development Fund Project of Shenzhen (Grant No. JCYJ20130402114702124).

\section{References}

1. Amin MB, Amin MB, Tamboli P, Javidan J, Stricker H, de-Peralta Venturina M, Deshpande A and Menon M: Prognostic impact of histologic subtyping of adult renal epithelial neoplasms: an experience of 405 cases. Am J Surg Pathol 26: 281-291, 2002.

2. Brisigotti M, Cozzutto C, Fabbretti G, Sergi C and Callea F: Metanephric adenoma. Histol Histopathol 7: 689-692, 1992.

3. Schmelz HU, Stoschek M, Schwerer M, Danz B, Hauck EW, Weidner W and Sparwasser C: Metanephric adenoma of the kidney: case report and review of the literature. Int Urol Nephrol 37: 213-217, 2005.

4. Liniger B, Wolf RW, Fleischmann A and Kluwe W: Local resection of metanephric adenoma with kidney preservation. J Pediatr Surg 44: E21-E23, 2009.

5. Kohashi K, Oda Y, Nakamori M, Yamamoto H, Tamiya S, Toubo T, Kinoshita Y, Tajiri T, Taguchi T and Tsuneyoshi M: Multifocal metanephric adenoma in childhood. Pathol Int 59: 49-52, 2009.

6. Bastide C, Rambeaud JJ, Bach AM and Russo P: Metanephric adenoma of the kidney: clinical and radiological study of nine cases. BJU Int 103: 1544-1548, 2009.

7. Yoshioka K, Miyakawa A, Ohno Y, Namiki K, Horiguchi Y, Murai M, Mukai M and Tachibana M: Production of erythropoietin and multiple cytokines by metanephric adenoma results in erythrocy tosis. Pathol Int 57: 529-536, 2007.

8. McNeil JC, Corbett ST, Kuruvilla S and Jones EA: Metanephric adenoma in a five-year-old boy presenting with chyluria: case report and review of literature. Urology 72: 545-547, 2008.

9. Jinzaki M, Tanimoto A, Mukai M, Ikeda E, Kobayashi S, Yuasa Y, Narimatsu Y and Murai M: Double-phase helical CT of small renal parenchymal neoplasms: correlation with pathologic findings and tumor angiogenesis. J Comput Assist Tomogr 24: 835-842, 2000.

10. Patel NP, Geisinger KR, Zagoria RJ and Bergman S: Fine needle aspiration biopsy of metanephric adenoma: a case report. Acta Cyto1 53: 327-331, 2009.

11. Olgac S, Hutchinson B, Tickoo SK and Reuter VE: Alpha-methylacyl CoA racemase as a marker in the differential diagnosis of metanephric adenoma. Mod Pathol 19: 218-224, 2006.

12. Rakheja D, Lian F, Tomlinson GE, Ewalt DH, Schultz RA and Margraf LR: Renal metanephric adenoma with previously unreported cytogenetic abnormalities: case report and review of the literature. Pediatr Dev Pathol 8: 218-223, 2005.

13. Renshaw AA, Freyer DR and Hanlners YA: Metastatic metanephric adenoma in a child. Am J Surg Pathol 24: 570-574, 2000.

14. Drut R, Drut RM and Ortolani C: Metastatic metanephric adenoma with foci of papilary carcinoma in a child: a combined histologic, immunohistochemical, and FISH study. Int J Surg Pathol 9: 241-247, 2001.

15. Pins MR, Jones EC, Martul EV, Kamat BR, Umlas J and Renshaw AA: Metanephric adenoma-like tumors of the kidney: report of 3 malignancies with emphasis on discriminating features. Arch Pathol Lab Med 123: 415-20,1999. 